TITLE:

\title{
A study on performance improvements of HTS coil
}

$\operatorname{AUTHOR}(S)$ :

Yamaguchi, M; Honma, A; Ishiguri, S; Fukui, S; Muta, I; Nakamura, T

\section{CITATION:}

Yamaguchi, M ... [et al]. A study on performance improvements of HTS coil. IEEE TRANSACTIONS ON APPLIED SUPERCONDUCTIVITY 2003, 13(2): 1848-1851

\section{ISSUE DATE:}

2003-06

URL:

http://hdl.handle.net/2433/39946

\section{RIGHT:}

(c)2003 IEEE. Personal use of this material is permitted. However, permission to reprint/republish this material for advertising or promotional purposes or for creating new collective works for resale or redistribution to servers or lists, or to reuse any copyrighted component of this work in other works must be obtained from the IEEE. 


\title{
A Study on Performance Improvements of HTS Coil
}

\author{
Mitsugi Yamaguchi, Atsushi Honma, Shinichi Ishiguri, Satoshi Fukui, Itsuya Muta, and Takatsune Nakamura
}

\begin{abstract}
It is of great concern for magnet designers to address their critical currents at a given field and bore size. In the case of high temperature superconducting magnets wound with BSCCO Ag-sheathed tapes, they have a strong magnetic anisotropy and therefore the critical current of magnets defined by an average voltage drop over an entire coil cannot be easily determined like low temperature superconducting magnets. An analytical way to obtain the critical current of HTS magnets is described by means of the electric and magnetic field analyzes based on the measured data of BSCCO Ag-sheathed tapes. A performance improvement of HTS magnet is studied by pursuing an optimum coil cross section.
\end{abstract}

Index Terms-Ag-sheathed Bi-2223 tape, critical current, flux density, magnetic anisotropy.

\section{INTRODUCTION}

$\mathbf{M}$ ORE applications of high temperature superconducting (HTS) magnets are expected from the point of view of attractive technical potentialities in accordance with the performance improvement of Bi-HTS tapes. It seems likely that the feasible applications in near future will be to MRI, NMR, energy storage device, reactor type fault current limiter [1], magnetic separator, etc.

The critical current of the Bi-HTS tape is governed by cooling temperature, magnetic field and its angle to Bi-HTS tape. Therefore, the exact coil performance cannot be easily evaluated at the coil design stage unless the above factors, in particular field inclination is taken into account over the coil cross section.

The cause of deterioration of coil critical current is due mainly to the perpendicular component of magnetic flux against HTS tapes. Attaching an iron, an iron yoke, employing side coils, etc. can achieve the alleviation of this problem [2]-[5]. However, it is more pertinent to manage radial flux reduction first of all by the coil itself. The auxiliary devices will be attached after this, if necessary.

A study on coil optimization of a rectangular cross section providing less volume was made by removing the large electric field portion, thus producing a stepped cross-section. For this purpose, fitting equations concerning the critical current and $n$ value of Bi-HTS tapes were derived from the short sample test data. The critical current characteristic of the rectangular cross section coil was measured and compared with analyzes based on fitting equations, providing a relatively good agreement.

Manuscript received August 6, 2002.

M. Yamaguchi, A. Honma, S. Ishiguri, and S. Fukui are with Niigata University, Niigata 950-2181, Japan (e-mail: yama@gs.niigata-u.ac.jp; honma@yamakena.gs.niigata-u.ac.jp; ishiguri@yamakena.gs.niigata-u.ac.jp; fukui@eng.niigata-u.ac.jp).

I. Muta and T. Nakamura are with Kyoto University, Kyoto 606-8501, Japan (e-mail: muta@kuee.kyoto-u.ac.jp; tk_naka@kuee.kyoto-u.ac.jp).

Digital Object Identifier 10.1109/TASC.2003.812923

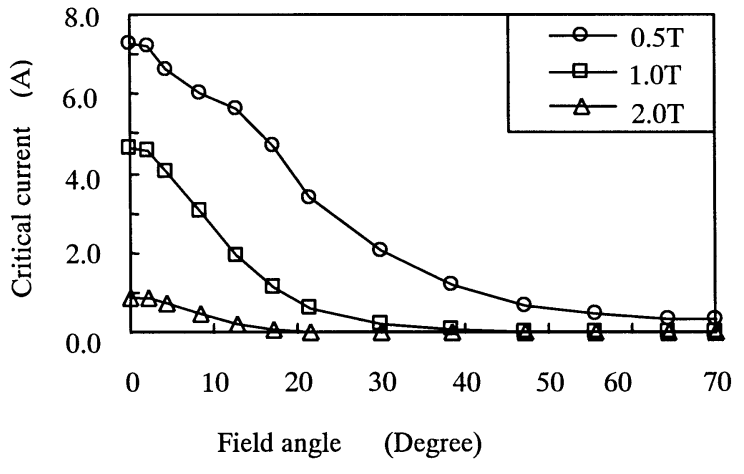

Fig. 1. Critical current characteristics of Bi-2223/Ag tape.

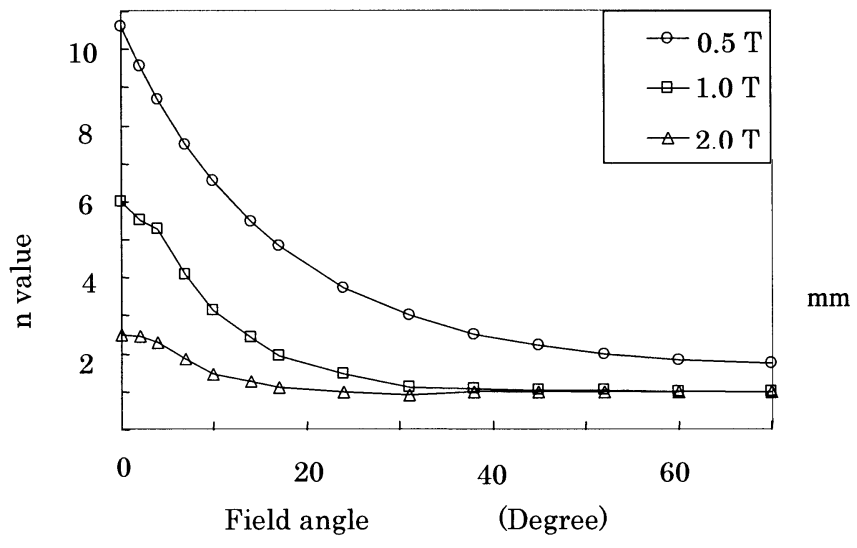

Fig. 2. $N$ value characteristics of $\mathrm{Bi}-2223 / \mathrm{Ag}$ tape.

The coil size optimization was achieved by both the field analyzes of the coil and the fitted characteristic equations of Bi-HTS tapes. As a result, the critical current of the optimized coil was improved by about $16.5 \%$ and the stored energy per unit volume by about $22.5 \%$.

\section{Fitting EQuations OF Bi-2223/Ag TAPE}

The magnetic anisotropic characteristics of $\mathrm{Bi}-2223 / \mathrm{Ag}$ tape at the field of $0.5 \mathrm{~T}$ to $2.0 \mathrm{~T}$ were measured at liquid nitrogen temperature of $77 \mathrm{~K}$ as a function of magnetic flux angle of 0 to 70 degrees to a tape. HTS tapes have 55 filaments and the silver ratio of 2.8. The width and the thickness of the tape are 3.20 $\mathrm{mm}$ and $0.21 \mathrm{~mm}$ respectively. The measured characteristics of tapes, namely the critical current and $n$ value are indicated in Figs. 1 and 2.

The current carrying characteristics of $\mathrm{Bi}-2223 / \mathrm{Ag}$ tapes is expressed by,

$$
V=V_{0}\left(\frac{I}{I_{c}}\right)^{n}
$$


where, $V_{0}$ corresponds to the flux flow voltage at the current of $I_{c}$. A critical current $I_{c}$ and an $n$ value are derived from the measured current voltage characteristics of the $\mathrm{Bi}-2223 / \mathrm{Ag}$ tape. Hereafter, $I_{c}$ is defined by $0.5 \mu \mathrm{V} / \mathrm{cm}$.

The fitting equations of $I_{c}$ and $n$ value at the temperature of $77 \mathrm{~K}$ are obtained from the above test results as a function of field $B$ and its angle $\theta$ such that,

$$
\begin{aligned}
I_{c}(B, \theta)= & 21.2 \times \exp \{-f(\theta) \times B\} \\
f(\theta)= & 2.02 \times 10^{-8} \times \theta^{5}-2.95 \times 10^{-6} \times \theta^{4} \\
& +1.04 \times 10^{-4} \times \theta^{3}+1.09 \times 10^{-3} \times \theta^{2} \\
& +0.03 \times \theta+1.70 \\
n(B, \theta)= & 16.8 \times \exp \{-g(\theta) \times B\} \\
g(\theta)= & -1.81 \times 10^{-9} \times \theta^{5}+5.10 \times 10^{-7} \times \theta^{4} \\
& -4.87 \times 10^{-5} \times \theta^{3}+1.29 \times 10^{-3} \times \theta^{2} \\
& +0.06 \times \theta+0.97
\end{aligned}
$$

\section{Minimum CoIL Volume}

It is well known that the central field of a solenoid coil $B_{0}$ is given by the next equation using Fabry Factor $F(\alpha, \beta)$.

$$
B_{0}=a_{1} i \lambda F(\alpha, \beta) \times 10^{-6}
$$

where, $i$ is a current density of conductors and $\lambda$ a packing factor of conductors in the coil winding. $F(\alpha, \beta), \alpha$ and $\beta$ are represented respectively as below.

$$
\begin{aligned}
F(\alpha, \beta) & =\frac{4 \pi}{10} \beta \ln \frac{\alpha+\sqrt{\alpha^{2}+\beta^{2}}}{1+\sqrt{1+\beta^{2}}} \\
\alpha & =\frac{a_{2}}{a_{1}} \\
\beta & =\frac{b}{a_{1}}
\end{aligned}
$$

where $a_{1}, a_{2}$ and $b$ are inner radius, outer radius and a halflength of a coil, respectively. The volume of a coil is expressed by the following equation and therefore the coil configuration providing the minimum coil volume can be obtained on the condition of a given constant Fabry Factor $F(\alpha, \beta)$, namely a constant central field.

$$
V=2 \pi a_{1}^{3} \beta\left(\left(\frac{K^{2}-\beta^{2}}{2 K}\right)^{2}-1\right)
$$

where, $K$ is described by,

$$
K=\left(1+\sqrt{1+\beta^{2}}\right) \exp \left(\frac{10 F(\alpha, \beta)}{4 \pi \beta}\right)
$$

and $\alpha$ and $\beta$ are related by the following equation.

$$
\alpha=\frac{K^{2}-\beta^{2}}{2 K} \text {. }
$$

Thus, the minimum coil volume can be obtained by the above equations for a given $F(\alpha, \beta)$.

Usually, an operation current of low temperature superconducting (LTS) magnets has an appropriate margin against the
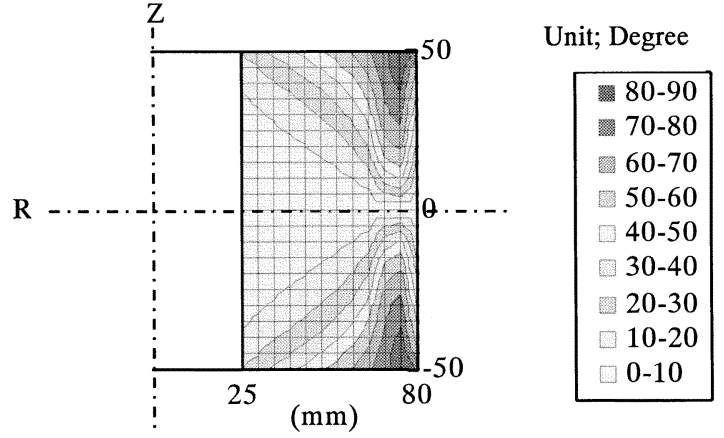

Fig. 3. Field angle distribution inside the HTS coil.

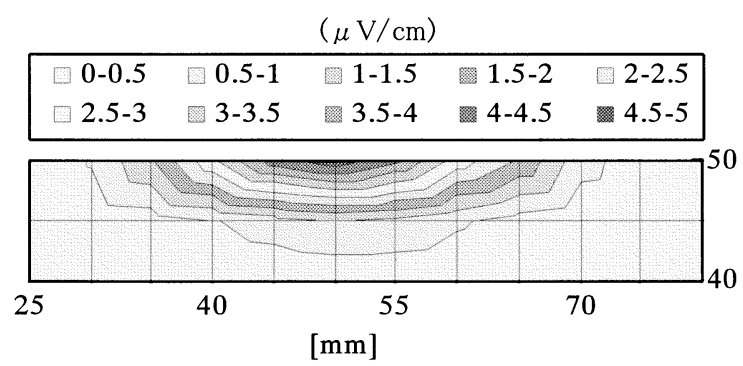

Fig. 4. Electric field distribution at the coil edge $\left(i=I_{c}\right)$.

critical current of short sample test. This determination process is mainly governed by the maximum field of a coil at the operation. On the other hand in the case of an HTS magnet, the high electric field appears at the middle portion of a coil edge, not at the coil bore due to the magnetic anisotropy. Accordingly, the critical current of a magnet is calculated on the basis of the average voltage drop per unit length in many cases, namely $\mu \mathrm{V} / \mathrm{cm}$ over the entire coil. Therefore, it is conceivable that the performance of magnets will be able to be improved by removing high electric field portions from the original rectangular cross section of a coil.

In order to define the critical current of a coil based on the average electrical field, the electrical field distribution characteristics need to be first addressed as a function of flowing currents at the coil cross section. This one can be calculated with the aid of fitting equations described above together with magnetic field analyzes of the coil.

Fig. 3 illustrates the calculated result of the distribution of magnetic field angle in the case of a minimum volume coil described later. This pattern of course differs more or less depending on the exact coil size and coil aspect ratio. It is evident from this flux angle distribution that magnetic fluxes intersect HTS tapes with sharper angles as they approach to the coil edge, though the magnetic field in general decreases. It is worthy of noting that the electrical field is governed by not only the flux angle but also the magnetic field and thus a high electrical field portion appears at the coil edge as shown in Fig. 4. The calculated result of current voltage characteristics of this coil is shown in Fig. 6, as described later.

\section{EVAluATION OF HTS COIL PERFORMANCE}

The effectiveness of a simulation method was confirmed by a model coil sketched in Fig. 5. Major dimensions of the model 


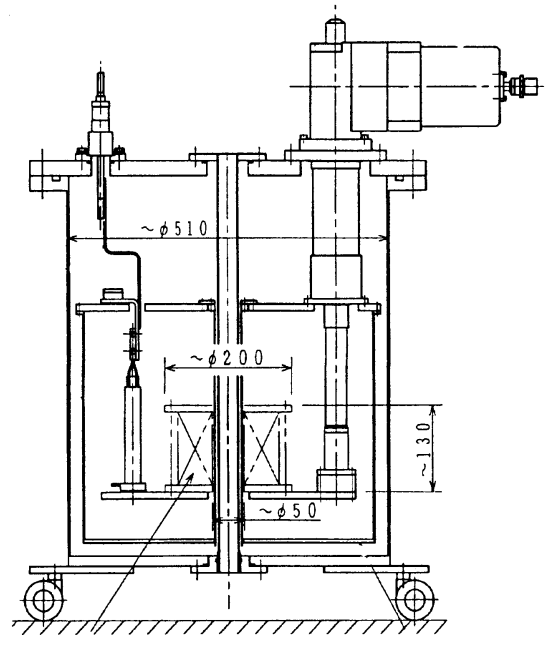

Fig. 5. Test device of HTS coil.

TABLE I

SPECIFICATION OF HTS COIL

\begin{tabular}{cc}
\hline \hline number of turns & 7365 \\
inner diameter $(\mathrm{mm})$ & 50 \\
outer diameter $(\mathrm{mm})$ & 160 \\
height $(\mathrm{mm})$ & 100 \\
& \\
critical current at $77 \mathrm{~K}(\mathrm{~A})$ & 6.70 \\
maximum field $(\mathrm{T})$ & 0.45 \\
number of double pancakes & 15 \\
inductance $(\mathrm{H})$ & 2.86 \\
\hline \hline
\end{tabular}

coil were determined in accordance with the idea of the minimum coil volume discussed in III. A coil with a bore of $50 \mathrm{~mm}$ and a height of $100 \mathrm{~mm}$ is composed of 15 double pancakes and total turns of 7365 are wound with aforementioned Bi-2223/Ag tapes. The specification of the model coil is listed in Table I. The refrigerator keeps the radiation shield at $77 \mathrm{~K}$ and can cool the magnet down to $10 \mathrm{~K}$. The coil temperature is controlled with the aid of a heater. Thermally good conducting plates are inserted in the magnet in order to make the temperature gradient as small as possible. The current is supplied to the magnet through the power lead made of HTS bulk material.

The measured critical current and $n$ value of the coil are $6.7 \mathrm{~A}$ and 9.6 respectively at the temperature of $77 \mathrm{~K}$. The distribution of the flux angle was calculated and the flux density was analyzed over the magnet cross section at the coil critical current of $6.7 \mathrm{~A}$. The maximum radial field of $0.16 \mathrm{~T}$ appears at the middle of the coil edge, while the maximum axial field of $0.45 \mathrm{~T}$ at the center of the bore. The current voltage characteristics of HTS coil were analyzed by using (1)-(5) described above. Fig. 6 shows analyzed results of the current voltage characteristics of the HTS coil together with the test result. The difference between the measured critical current and the analysis is $0.45 \mathrm{~A}$. It seems likely that this simulation is reasonable since a possible performance degradation due to the winding of $\mathrm{Bi}-2223 / \mathrm{Ag}$ tape and an inevitable nonuniformity of BSCCO Ag-sheathed tape properties with length are not taken into account.

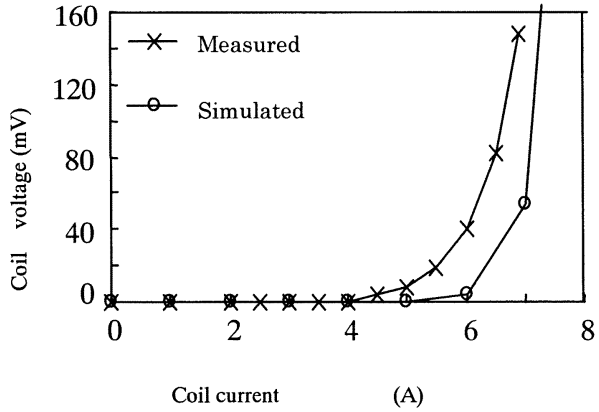

Fig. 6. Current voltage characteristics of HTS coil.

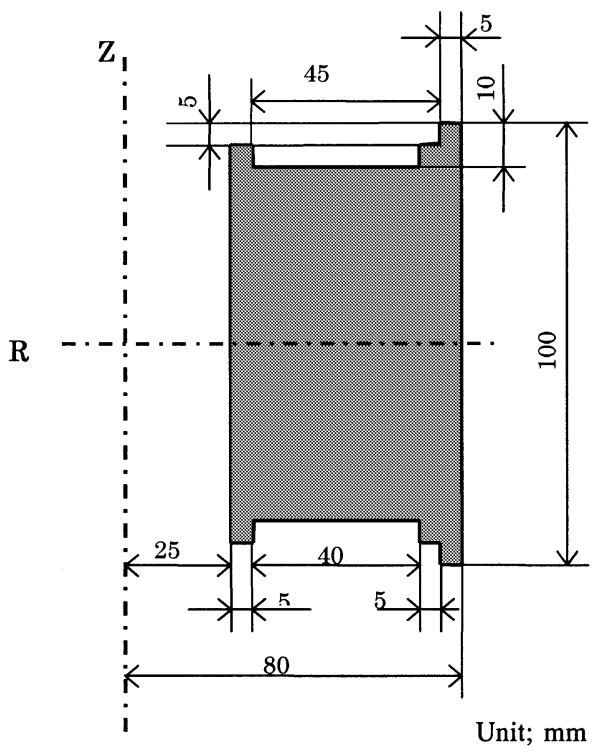

Fig. 7. Optimized coil cross section.

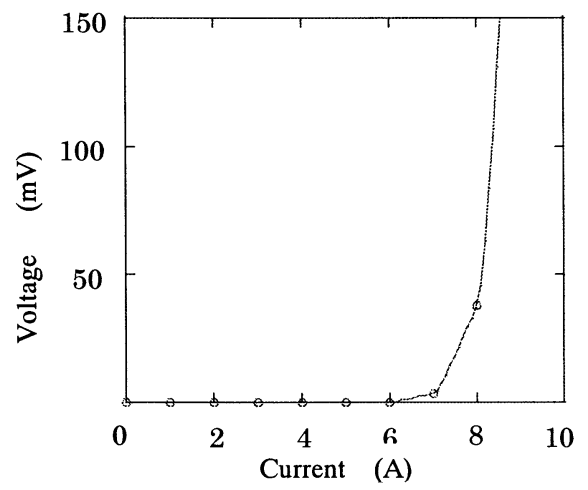

Fig. 8. Current voltage characteristics of optimized coil.

\section{Optimization of CoIL Cross Section}

The high electric field portions appear at the central portion of the coil edge due to the sharply curved magnetic flux lines. Therefore, the removal of HTS tapes at high electric field portions decreases the averaged electric field of the coil. Thus, the original coil configuration with the rectangular cross section is optimized by removing HTS tapes at the region of electric field higher than $0.5 \mu \mathrm{V} / \mathrm{cm}$, as depicted in Fig. 7.

In this new optimized coil, about $16 \%$ of cross section was taken out, producing the stepped shape at the coil edge. Fig. 8 indicates the current voltage characteristics of the optimized coil. 


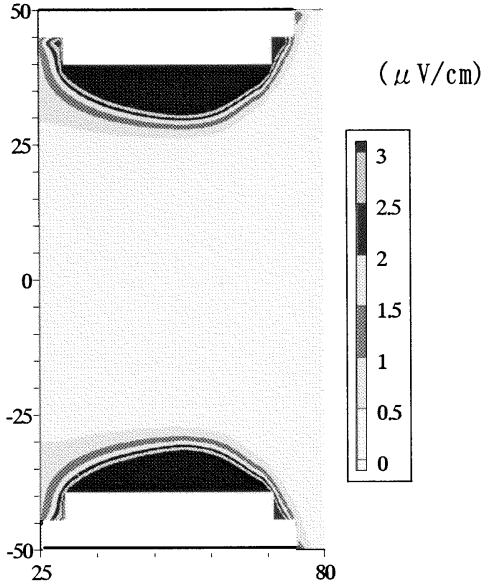

Fig. 9. Field distribution of optimized coil $\left(i=I_{c}\right)$.

TABLE II

COMPARISON OF COIL PERFORMANCES

\begin{tabular}{ccc}
\hline \hline & $\begin{array}{c}\text { Rectangular } \\
\text { Cross Section }\end{array}$ & $\begin{array}{c}\text { Optimized } \\
\text { Cross Section }\end{array}$ \\
\hline Number of turns & 7365 & 6160 \\
Central field (T) & 0.45 & 0.46 \\
Maximum field (T) & 0.48 & 0.49 \\
Critical current (A) & 7.15 & 8.33 \\
$n$ value & 20.48 & 19.2 \\
Inductance (H) & 2.86 & 2.17 \\
Stored energy (J) & 73.1 & 75.1 \\
Stored energy (J/cm $\left.{ }^{3}\right)$ & 0.040 & 0.049 \\
Flux flow loss at Ic (W) & 0.868 & 0.856 \\
\hline \hline
\end{tabular}

The critical current of the new coil is increased by $16.5 \%$ from 7.15 A to 8.33 A by means of the above optimization process. Fig. 9 displays the electric field distribution of the optimized coil at the critical current.

\section{Performance Comparison of Each CoIL}

Table II compares the optimized coil with the original rectangular cross section coil. The critical current became $16.5 \%$ larger notwithstanding that $16.3 \%$ of BSCCO Ag-sheathed tapes were not in use and the central field also increased. The new configuration coil can store the energy of $75.1 \mathrm{~J}$, about $2.6 \%$ larger than the original one.

It is worthwhile to note that the stored energy per unit winding volume of the optimized coil is about $22 \%$ larger than the rectangular shape coil, while the flux flow loss is almost the same.

These performance improvements were achieved by the first step optimization. It is conceivable that HTS coil performance will be improved further, though it might not be so conspicuous as the first one, if the similar optimization process is proceeded.

\section{CONCLUSIONS}

BSCCO Ag-sheathed tapes have strong magnetic anisotropic properties and accordingly generate a high electric field region at the coil edge portion. The coil optimization, which will produce a larger critical current, is made by removing the high electric field portion from a rectangular cross section coil based on measured magnetic anisotropic data of Bi/Ag-sheathed tape. The optimized coil can store the more energy with fewer amounts of superconducting materials.

\section{REFERENCES}

[1] M. Yamaguchi, S. Fukui, T. Satoh, Y. Kaburaki, T. Horikawa, and T. Honjo, "Performance of dc reactor type fault current limiter using high temperature superconducting coil," IEEE Trans. on Applied Superconductivity, vol. 9, no. 2, pp. 940-943, 1999.

[2] T. Nakamura, S. Tsuchiya, A. Fujio, T. Hoshino, I. Muta, and M. Yamaguchi, "Angular dependence of E-J characteristics and dissipative properties in Bi-2223/Ag tape," Supercond. Sci. and Technol., vol. 15, pp. 230-235, 2002.

[3] J. Pitel, P. Kovac, and I. Husek, "Calculation of the critical currents of $\mathrm{Bi}(2223) / \mathrm{Ag}$ tapes and coils with reduced anisotropy in $I_{c}(B)$ characteristic-effects of different proportional representations of the filaments orientated parallel and perpendicularly to the tape surface," Physica C, vol. 330, pp. 130-140, 2000

[4] S. Noguchi, M. Yamashita, H. Yamashita, and A. Ishiyama, "An optimal design method for superconducting magnets using HTS tape," IEEE Trans. on Applied Superconductivity, vol. 11, no. 1, pp. 2308-2311, 2001

[5] W. Nah, J. Kang, S. Choi, I. Park, J. Joo, Y. Kwon, S. Oh, K. Ryu, J. Paasi, and J. Lehtonen, "Numerical calculation of critical current in Bi-2223 stacked tapes," IEEE Trans. on Applied Superconductivity, vol. 11, no. 1, pp. 3908-3911, 2001. 